\title{
Impact of Intervention with Co-Enzyme Q10 on Homocysteine Levels of Cardiac Patients with Established Angiographic Evidence

\author{
Mathur Ritu* and Mathur Manika
}

Department of Food Science and Nutrition, MDS University, Ajmer, Rajasthan 305009, India

Received: August 30, 2014; Accepted: November 11, 2014; Published: November 27, 2014

*Corresponding author: Mathur Ritu, Department of Food Science and Nutrition, MDS University, Ajmer, Rajasthan 305009, India, E-Mail: drritumathur@gmail.com

\begin{abstract}
Nutritionists and Health care professionals, epidemiologists and International agencies like WHO have been sounding an alarm on the remarkable increase in the incidence of Cardiovascular Diseases (CVD) for the past 15 years all around the world. It has been estimated that by 2020, CVD will be the largest cause of disability and death in India, which is the major reason why clinical research in this field is extremely relevant. The present study was undertaken to assess the nutritional status of twenty subjects suffering from coronary artery disease, cardiomyopathy, hypertension, mitral valve prolapse or coronary revascularisation with established angiographic evidence. Complete nutritional status assessment was done which included anthropometric, dietary and biochemical estimations. These subjects were then given $100 \mathrm{mg}$ soft gel capsules of Coenzyme Q10 for a period of 60 days (this Intervention Trial was duly approved by the Institutional Ethics Committee). The study revealed that although the Body Mass Index (BMI) of the subjects was in the normal range they had high Waist/Hip Ratios indicating that central obesity was one of the risk factors for their CVD. The diets were found to be high in total fats and saturated fats, low in protein, fibre, and omega 3 fatty acids. The biochemical profile showed that the subjects had high levels of fasting and PP blood sugar and Lipoprotein A. homocysteine levels were found to be very high as compared to the normal values ( $p \leq$ 0.01 ) in all the cardiac subjects prior to intervention. The subjects were asked to give their consent for the 60 days Intervention Trial with $100 \mathrm{mg}$ of Coenzyme Q10 on Informed Consent Sheets. After Intervention all the Biochemical tests were conducted again. Results revealed that the impact of Coenzyme Q10 in lowering blood Sugar, Cholesterol, TG, LDL and Lipoprotein A levels was not significant which may be due to a modest dose and short intervention period of 60 days. homocysteine is a new emerging and independent risk factor for CVD There was a significant reduction $(p \leq 0.01)$ in the blood homocysteine (Hcy) levels of the subjects (from $22.52 \pm 10.13$ to17.08 \pm 6.21 ). Hence in conclusion it can be said that Coenzyme Q10 is a rich, powerful and safe antioxidant with many beneficial therapeutic properties. Its supplements in the diet along with a diet which is rich in folic acid, both of which have a role in the correction of hyper homocysteinemia, can go a long way in the prevention of the global epidemic of CVD in the future times to come.
\end{abstract}

Keywords: Cardio vascular diseases; Saturated fats; Body Mass Index; Lipoprotein (a); homocysteine Coenzyme Q10

\section{Introduction}

Coronary Heart Disease (CHD) is a condition in which the walls of the arteries supplying blood to the heart muscle become thickened. This thickening which is caused by the development of lesions in the arterial wall is called atherosclerosis; the lesions are called plaques. It can restrict the supply of blood in the heart muscle (myocardium) and may manifest to the patient as chest pain on exertion (angina) or breathlessness on exertion. CHD is also referred to as Ischaemic Heart Disease (IHD). A heart attack (also called Myocardial Infarction) is when part of the heart muscle is damaged or dies because it isn't getting enough blood from the coronary arteries. Heart attacks usually result from a blockage in the coronary arteries. This blockage is most likely to be caused by a blood clot that forms where an atherosclerotic plaque has cracked or ruptured [1]. An estimated 17 million people die from Cardiovascular Disease (CVDs) particularly heart attacks and strokes every year, thus making stroke the second leading cause of death. Projections to the year 2020 indicate an increase in the number of CVD cases, majority of such cases will be from developing countries including India [2]. Raised blood pressure is the most important CVD risk factor, attributing to one half of the coronary heart diseases and approximately two third of the cerebrovascular diseases because it exerts excessive pressure on the interior wall of arteries, thereby damaging internal endothelial lining of the blood vessels and at the damaged sites [3].

The risk factors of CVD include age, sex, hypertension, heredity and faulty dietary habits, smoking, obesity, diabetes, elevated serum LDL cholesterol and reduced HDL cholesterol levels. Emerging independent risk factors include elevated serum concentration of Lipoprotein (a), C-reactive proteins, Fibrinogen, ruminant lipoproteins and homocysteine [4-6].

The cornerstone of CHD prevention is lifestyle modification. Dietary intervention studies support that restricting saturated fat and cholesterol and increasing the intake of essential fatty acids, especially n-3 fatty acids, reduces CHD risk. The available data indicate that there should be a recommendation to use animal, 
dairy and hydrogenated fats, tropical oils, egg yolks and sugars sparingly and to encourage the use of vegetables, fruit and whole grains for the prevention from CHD.

\section{Homocysteine and risk of CVD}

In 1968 a Harvard researcher named Dr. Kilmer Mc Cully [7] proposed that homocysteine (Hcy) might be a significant and independent risk factor for heart disease. Since inflammation and oxidative stress are critical to Hcy induced vascular damage, the improvement of endothelial dysfunction and the inhibition of mononuclear cell activation by anti-inflammatory and/or antioxidative drugs/agents may serve as the potential therapeutic strategy for hyper homocysteinemia related CVD. Based on secular trends in the United States and the United Kingdom, the greater reduction in stroke mortality from 1990 through 2002 in the U.S. have been tentatively attributed to the introduction of folic acid fortification [8]. A previous meta-analysis of published results from some of the folic acid trials, reported that folic acid could reduce the risk of stroke [9]. Under normal metabolic circumstances, there is a strict balance between Hcy formation and elimination. Under condition of reduced or total loss of activity of Hcy metabolizing enzyme due to mutation in corresponding gene, the metabolic balance is disturbed resulting into hyper homocysteinemia [10]. Human studies on folic acid, homocysteine and endothelial function are reported. Study proposed that folic acid in high doses may have beneficial effects on endothelial function, which are independent of Hcy lowering [11]. In countries with a low folate intake in particular the Hcy concentration was higher than ideal [12]. Once the relationship between Hcy level and heart diseases is judged to be causal, it follows that reducing serum Hcy levels will reduce the risk of IHD. This can be achieved by increasing the consumption of the vitamin folic acid. A folic acid supplement of $0.4 \mathrm{mg} / \mathrm{dl}$ has been shown to reduce average Hcy levels in middle aged patients by $19 \mu \mathrm{mol} / \mathrm{L}$ [13].

\section{Role of Antioxidants in CVD}

Inflammation triggered by oxidative stress is the cause of much, perhaps even most chronic human disease including human aging. The oxidative stress originates mainly in the mitochondria from reactive oxygen and reactive nitrogen species and can be identified in most of the key steps in the pathophysiology of atherosclerosis and the consequential clinical manifestations of cardiovascular disease [14]. Over the years an oxidative stress hypothesis is supported by epidemiologic and observational evidence that encouraged belief in the use of antioxidants [15]. Antioxidants in fruits and vegetables are important in inhibiting oxidative mechanism that lead to various degenerative diseases including CVD [16]. Numerous clinical trials have evaluated the use of nutritional supplements such as beta-carotene, selenium, vitamin $\mathrm{C}$ and vitamin $\mathrm{E}$ in the prevention of CHD and stroke [17]. Flavanols appear to be the substances in cocoa that are responsible for lowering B.P. and boosting heart health [18]. Coenzyme Q10 is one such antioxidant which has been found to have a beneficial impact both in the prevention and treatment of cardiovascular diseases.

\section{Coenzyme Q10 and CVD}

Coenzyme Q10 (CoQ10) or ubiquinone is essentially a vitamin or vitamin - like substance. It is present naturally in foods and sometimes is also synthesized in the body. CoQ10 like-wise is found in small amounts in a wide variety of foods and is synthesized in all tissues. Normal blood and tissue levels of CoQ10 have been well established by numerous investigators around the world. Significantly decreased levels of CoQ10 have been noted in a wide variety of diseases in both animal and human studies. CoQ10 deficiency may be caused by insufficient dietary CoQ10, impairment in CoQ10 biosynthesis, excessive utilization of CoQ10 by the body, or any combination of the three. Decreased dietary intake is presumed in chronic malnutrition and cachexia [19]. CoQ10 has received particular attention in the prevention \& treatment of various forms of CVDs. It is highly effective in preventing the oxidation of low density lipoprotein cholesterol (LDL) that leads to atherosclerosis. Supplementation with Co Q10 has also been found beneficial in patients with chronic stable angina, mitral value prolapse \& irregular heartbeat [20] and in patients suffering from Hypertension [21]. Levels of Co Q10 tend to be lower in people with high cholesterol. In addition, certain cholesterol lowering drugs called statins appear to deplete natural levels of Co Q10 in the body. Taking Co Q10 supplements can correct the deficiency caused by statin medications without affecting the medication's positive effects on cholesterol levels. Clinical research indicates that introducing Co Q10 prior to heart surgery, including bypass surgery and heart transplantation, can reduce danger caused by free radicals, strengthens heart function and lower the incidence of irregular heart beat during the recovery phase [22]. The recommended daily dosage for health maintenance is $30 \mathrm{mg}$, however considerably higher amounts are required in the treatment of the various diseases for which supplementation has been found beneficial. Co Q10 should be taken with a meal containing some fat or even better in combination with soy or vegetable oil which enhances its absorption quite substantially [23]. For adults 19 years \& older; the recommended dose for Co Q10 supplementation is $30-200$ $\mathrm{mg}$ daily. Studies have shown that supplementation with as little as $100 \mathrm{mg} / \mathrm{dl}$ for 12 months results in better pumping capacity, increased muscle strength \& improved breathing [24].

\section{Safety of CoQ10}

The body readily absorbs CoQ10 supplements \& no toxic effects have been reported for daily doses as high as $300 \mathrm{mg}$. The safety of Co Q10 however has not been established in pregnancy \& lactation, so caution is advised here until more data becomes available [25]. Adverse effects with Co Q10 are rare. On average, mild gastrointestinal discomfort is reported in less than 1 percent of patients in clinical trials [26].

Hence the present study was undertaken for the purpose of assessment of Nutritional Status of twenty cardiovascular disease patients with established angiographic evidence in the city of Ajmer (Rajasthan, India) and to assess the impact of intervention with Coenzyme Q10 in these patients. 
Table 1: Anthropometric Data of the Cardiac Subjects.

\begin{tabular}{|c|c|c|c|c|}
\hline & Height (Ft) & Weight (kg) & BMI (kg/m $\mathbf{m}^{2}$ & W/H ratio \\
\hline Mean & 5.57 & 66.2 & 23.69 & 0.93 \\
\hline S.D (n= 20) & \pm .23 & \pm 4.92 & \pm 2.01 & \pm 0.02 \\
\hline
\end{tabular}

Table 2: Biochemical Data of Cardiac Subjects- Prior to Intervention \& Post Intervention with Co enzyme Q10.

\begin{tabular}{|c|c|c|c|c|c|c|c|c|c|c|}
\hline & & \begin{tabular}{|c|} 
Sugar \\
fasting \\
(mg/dl)
\end{tabular} & $\begin{array}{c}\text { Sugar PP } \\
\text { (mg/dl) }\end{array}$ & Hcy $\mu \mathrm{mol} / \mathrm{L}$ & $\mathrm{Lp}(\mathrm{a})(\mathrm{mg} / \mathrm{dl})$ & $\begin{array}{l}\text { Cholesterol(mg/ } \\
\text { dl) }\end{array}$ & TG(mg/dl) & VLDL(mg/dl) & HDL(mg/dl) & LDL(mg/dl) \\
\hline $\begin{array}{l}\text { Normal } \\
\text { Values }\end{array}$ & & $60-110$ & $<145$ & $5-15$ & $0-30$ & $<200$ & $50-160$ & $10-50$ & $35-80$ & $0-100$ \\
\hline \begin{tabular}{|c|} 
Pre \\
Intervention \\
$(n=20)$ \\
\end{tabular} & $\begin{array}{l}\text { Mean } \pm \\
\text { S.D. }\end{array}$ & $\begin{array}{c}118.45 \pm \\
55.88\end{array}$ & $\begin{array}{c}160.44 \pm \\
76.02\end{array}$ & $\begin{array}{l}22.52^{* *_{ \pm}} \\
10.13\end{array}$ & $30.0 \pm 28.27$ & $170.1+50.83$ & $\begin{array}{c}132.55 \pm \\
60.91\end{array}$ & $26.51 \pm 12.18$ & $41.6 \pm 7.6$ & $\begin{array}{l}73.85 \pm \\
23.48\end{array}$ \\
\hline \begin{tabular}{|c|} 
Post \\
Intervention \\
$(\mathrm{n}=20)$
\end{tabular} & $\begin{array}{l}\text { Mean } \pm \\
\text { S.D. }\end{array}$ & $\begin{array}{c}119.45 \\
\mathrm{~ns}_{+}+54.33\end{array}$ & $\begin{array}{c}165.01 \\
\mathrm{~ns}_{ \pm} 81.77\end{array}$ & $\begin{array}{l}17.08 * \pm \\
6.21\end{array}$ & $\begin{array}{l}31.05^{\mathrm{ns}} \pm \\
27.65\end{array}$ & $185.95^{\mathrm{ns}} \pm 48.15$ & $\begin{array}{c}139.5^{\mathrm{ns}} \pm \\
50.88\end{array}$ & $28.9^{\text {ns }} \pm 11.24$ & $\begin{array}{l}41.85^{\mathrm{ns}} \pm \\
8.55\end{array}$ & $\begin{array}{l}74.5^{\mathrm{ns}} \pm \\
21.16\end{array}$ \\
\hline
\end{tabular}

Table 3: Mean Nutrient Intake of the Cardiac Subjects Selected for Intervention and their Comparison with RDAs.

\begin{tabular}{|l|c|c|c|c|c|c|c|c|}
\hline & & $\begin{array}{c}\text { Energy } \\
\text { (Kcal) }\end{array}$ & $\begin{array}{c}\text { Protein } \\
(\mathbf{g m})\end{array}$ & $\begin{array}{c}\text { Fat } \\
\mathbf{( g m )}\end{array}$ & Fiber (gm) & $\begin{array}{c}\text { Sodium } \\
\text { (mg) }\end{array}$ & $\begin{array}{c}\text { P/S } \\
\text { Ratio }\end{array}$ & W3 Fatty Acid \\
\hline \multirow{3}{*}{$\begin{array}{l}\text { Men } \\
(\mathbf{n}=\mathbf{1 5})\end{array}$} & RDA & 2320 & 60 & 25 & 25 & 2092 & - & - \\
\cline { 2 - 9 } & Mean Intake & $1771.7^{* *}$ & $56.3^{\text {ns }}$ & $59.9^{* *}$ & $8^{* *}$ & $328^{* *}$ & 1.3 & 2.0 \\
\cline { 2 - 9 } & S.D. & 295.9 & 18.5 & 13.8 & 4.46 & 219.8 & 1.2 & 0.66 \\
\hline \multirow{2}{*}{$\begin{array}{l}\text { Women } \\
(\mathbf{n}=\mathbf{5})\end{array}$} & RDA & 1900 & 55 & 20 & 25 & 1902 & - & - \\
\cline { 2 - 9 } & Mean Intake & $1639.9^{\text {ns }}$ & $46.2^{*}$ & $106.6^{* *}$ & $5.6^{* *}$ & $244.38^{* *}$ & 1.2 & 1.9 \\
\cline { 2 - 10 } & S.D. & 348.8 & 8.74 & 56.7 & 0.78 & 137.34 & 0.77 & 0.54 \\
\hline
\end{tabular}

Values are mean \pm SD

**Significant at 0.01 levels

ns $=$ Not significant

\section{Materials and Methods}

This research study aimed at assessing the Nutritional Status of twenty cardiovascular disease patients which included complete Anthropometric assessment, Dietary assessment, Biochemical assessment and assessment of the impact of intervention with 100 mg Coenzyme Q10, a nutritional supplement and an antioxidant on patients with angiographic evidence of CVD.

\section{Experimental Design}

The study was divided into 3 phases:

Phase I: In this phase 20 subjects were selected, suffering from cardiovascular disease with established angiographic evidence. A complete Nutritional Status Assessment was done for these twenty selected subjects.

Phase II: The selected subjects were given $100 \mathrm{mg}$ soft gel capsules of Coenzyme Q10 to assess its impact on serum lipids, Lipoprotein and homocysteine levels and on the blood sugar levels.

Phase III: The impact of intervention with CoQ10 on the biochemical parameters of the subjects was assessed in this phase.

\section{Phase I}

Selection of subjects: For the intervention trial a total of 20 middle aged subjects (30-60 years)( men and women) were selected using purposive and convenience sampling method. Patients selected were diagnosed and confirmed to be suffering from any kind of cardiovascular disease with established angiographic evidence.

The criteria kept for selection was:

1. Patients (men \& women) suffering from coronary artery disease, cardiomyopathy, hypertension, mitral valve prolapse and coronary revascularisation with established angiographic evidence.

2. Pregnant and breastfeeding patients were not included in this study.

3. Women of childbearing potential but not using any form of effective method of contraception were included.

4. Patients who were willing to co-operate in the study.

5. Subjects residing in Ajmer city.

Anthropometry of all the twenty subjects were done. It included the following parameters - Weight, height, Waist Hip 
Ratio and Body Mass Index of the subjects. Different biochemical estimations of all the subjects were done which included - Blood Glucose Fasting, Post Prandial (PP) [27] Total Cholesterol [28], Triglycerides (TG) [29], HDL Cholesterol [30], LDL Cholesterol, VLDL Cholesterol, LDL/HDL ratio, Lipoprotein (a), and homocysteine (Immulite 1000 Analyzer).

The dietary intake data was assessed by collecting retrospective intake data and summarizing prospective intake data with the goal to determine the nutrient content of the food and the appropriateness of the intake for a particular individual. Information related to the dietary intake and food patterns of the subjects was collected through this questionnaire by a 24 hour recall method for three consecutive days $(24 \times 3=72$ hours recall) General information of all the twenty subjects was collected. General information consisted of all the personal information of the subjects, i.e. information regarding socio-economic status, education, type of family, number of family members, occupation etc. Two types of information on food intake were collected first about the qualitative aspects of food consumed i.e. kind of food eaten and the second about the food consumed in quantitative terms i.e. how much of food was taken. The 24 hour recall method was clubbed together with the weighment method to collect the dietary information. The different food items consumed were converted into their raw equivalents, categorised into the respective food groups and the average daily intake of energy, proximate principles, important minerals including sodium and fibre were calculated from the value per $100 \mathrm{gm}$ of edible portion given in the Food Composition Tables and compared to the RDA given by the ICMR for adults [31].

\section{Phase II: Intervention trial with CoQ10}

Procurement: For the intervention trial Coenzyme Q10 (100 mg) soft gel capsules were procured from Medizen Labs, Bangalore, India, for the purpose of authenticity and purity of the compound.

Intervention procedure: The intervention trial was conducted on these CVD patients with established angiographic evidence for a period of 60 days. The subjects were asked to take $100 \mathrm{mg}$ of CoQ10 i.e. one soft gel capsule everyday along with water, at the time of lunch. The Intervention Trial was duly approved and cleared by the Institutional Ethics Committee (IEC) which was constituted and which functioned as per the Indian Council of Medical Research (ICMR) Guidelines (2008) [32]. The IEC reviewed and approved all the ethical aspects of the study with respect to the welfare and safety of the subjects and the scientific soundness of the research work. The approval of the IEC was also communicated to the subjects participating in this research study. An Informed Patient Consent Form was given to individual subjects along with a coloured pamphlet regarding detailed scientific information about coenzyme Q10. This form was duly signed by each and every subject and only after receiving their written consent, the intervention trial began.

Phase III: Assessment of impact of CoQ10 on biochemical profile of the subjects
The impact of intervention with CoQ10 on the biochemical profile of all the subjects were assessed after a period of 60 days of intervention trial. The same biochemical tests were performed on all the subjects as in Phase I to observe the changes in their lipid levels.

\section{Statistical Analysis}

The results were statistically analysed by calculating the mean and standard deviations [16]. The $t$ test statistics was used for determining the difference between the means at 0.01 and 0.05 levels. The test of hypothesis for the existence of a linear relationship between two variables was conducted by determining the two tailed correlation coefficient [33].

\section{Results and Discussion}

This present piece of research was conducted to assess the Nutritional Status of twenty cardiovascular disease patients and to assess the impact of intervention with $100 \mathrm{mg}$ Coenzyme Q10 with angiographic evidence on these patients of CVD.

Anthropometric data of all the twenty subjects were measured prior to the intervention trial. The parameters included Height, Weight, Body Mass Index (BMI) and Waist/Hip ratio. The mean height of the subjects was found to be $5.57 \pm 0.23 \mathrm{ft}$.

A platform weighing balance was used to measure the weight of the subjects. The average weight of the subjects in the present study was $66.20 \pm 4.92 \mathrm{~kg}$. BMI of the subjects was found to be within the normal range i.e. $23.69 \pm 2.01$ (Normal Range - $20-25 \mathrm{~kg} / \mathrm{m}^{2}$ ). Waist hip ratio is also associated with obesity and increased risk of CVD. All the subjects $(n=20)$ had Waist/Hip ratio higher than normal $(0.93 \pm 0.02)$ indicating one of their possible risk factors for suffering from CVD. Regional fat distribution, particularly in the abdomen and hip, is considered important in development of CHD [34] as was seen in the present study also.

Different biochemical estimations of all the subjects $(n=20)$ was done twice, i.e. prior to intervention and after intervention with coenzyme Q10. Fasting blood samples were drawn for all the biochemical tests. Mean values were calculated and were then compared with the normal values. The Lp (a) of the subjects was found to be towards the higher side $(30 \pm 28.27 \mathrm{mg} / \mathrm{dl})$. Both blood sugar fasting and P.P. were found to be slightly greater than the normal values i.e. $118.45 \pm 55.88$ and $160.49 \pm 76.00$ $\mathrm{mg} / \mathrm{dl}$ respectively. Researchers have shown both Diabetes and Lipoprotein A levels to be associated with increased risk of CVD [4-6] as was seen in the present study. An elevated plasma level of the amino acid homocysteine is a significant and independent risk factor in the development of CHD [35]. Mean Hcy levels prior to intervention was found to be much higher $\left(22.25^{* *} \pm 10.05\right)$ than the normal values. The link between high levels of serum Hcy and atherosclerosis disease was seen in these subjects.

Lipid profile of all the subjects was also assessed prior to intervention. As shown in Table 2, the serum TG and cholesterol levels prior to intervention were found to be within the normal 
range. There is a strong inverse relationship between HDL concentration and coronary events. Low levels of HDL-C $(<40$ $\mathrm{mg} / \mathrm{dl}$ ) is an independent risk factor of CVD and raising HDL-C is a major treatment strategy for regressing atherosclerosis and enhancing CVD risk reduction [36]. HDL levels of the subjects prior to intervention was found to be $41.6 \pm 7.60 \mathrm{mg} / \mathrm{dl}(\mathrm{p}<0.01)$ LDL and VLDL values were found to be lying in the normal range i.e. $73.85 \pm 23.48 \mathrm{mg} / \mathrm{dl}$ and $26.51 \pm 12.18 \mathrm{mg} / \mathrm{dl}$ respectively.

To assess the impact of intervention with $100 \mathrm{mg}$ of CoQ10 soft gel capsules in the selected subjects of CVD $(n=20)$ with established angiographic evidence, the entire blood biochemical profile was repeated on completion of the intervention trial i.e. after the 60 days period. Results revealed that the impact of CoQ10 at the $100 \mathrm{mg}$ dose in lowering the fasting and PP blood sugar levels, cholesterol, TG, HDL, LDL, VLDL and Lp (a) was not significant when compared with the pre intervention values. However, Hcy which is a major emerging risk factor for CVD was found to be significantly lowered $(p \leq 0.05)$ following 60 days intervention trial. CoQ10 has been reported to have a beneficial effect in the prevention and treatment of various forms of CVD. [23].

Diet is a vital determinant of health and nutritional status of the people. Diets of all the 20 subjects were assessed and calculated. Energy, Protein, Fat, Fiber \& Sodium content of the diet was calculated and compared to the RDA given by the ICMR [31]. The energy and protein intake of subjects was lower than the RDA in both men and women. The results of the present study revealed that the fibre intake in both men and women was very low when compared with the RDA. It was $8 \pm 4.46 \mathrm{gm}$ and 5.6 \pm 0.78 in men and women respectively. Epidemiological studies have found high fibre diets to have beneficial effects in the prevention of CVD [37]. High fat intake bears direct correlation with BMI and lipid profile of the subjects. High dietary saturated fat has therefore been associated with increased risk of CVD. All the subjects were consuming a very high fat diet. When compared with the RDA, the values were found to be highly significant at $p$ $\leq 0.01$ level. The mean fat intake in men subjects was $59.9 \pm 13.8$ $\mathrm{gm}$, whereas in women subjects the fat intake was even higher than men $(106.6 \pm 56.7 \mathrm{gm})$.Increased consumption of dietary fat leads to an increase in body weight, waist circumference and mean systolic and diastolic blood pressure which are also associated with high intakes of sodium. Long term sodium reduction and potassium substitution may lead to a reduced risk of CVD. Data also revealed a low intake of omega 3 fatty acids in the diets of the selected subjects and the Polyunsaturated to Saturated fatty acid ratio was also low indicating that the subjects were at a high risk of developing CVD [Table 3].

Hence it can be concluded that faulty dietary practices reflected their impact on the anthropometric and biochemical profile of the cardiac subjects all of whom were suffering from some or the other kind of cardiovascular disease with angiographic evidence. A high fibre and a high Folic acid diet which is low in saturated fats, salt and cholesterol is recommended for the prevention of CVD. It can also be said that Coenzyme Q10 is a promising antioxidant with diverse therapeutic properties and a great degree of safety. Its role in preventing cardiovascular diseases and in the correction of hyper homocysteinemia can prove to be a milestone in lowering the incidence of this global, frightening and often fatal epidemic of CVD.

\section{References}

1. Krauss RM. Lipoprotein sub fractions and cardiovascular disease risk. Curr Opin Lipidol. 2010; 21(4): 305-311.

2. World Health Organisation. Non communicable diseases in South East Asia region: A profile. New Delhi World Health Organisation; 2002.

3. Libby P, Ridker PM, Maseri A. Inflammation and atherosclerosis. Circulation; Inflammation and atherosclerosis. 2002; 105: 1135-1143. doi: $10.1161 /$ hc0902.104353.

4. Kaptoge S, Angelantonio E, Lowe G, Pepys MB, Thompson SG, Collins $\mathrm{R}$, et al. C-reactive protein concentration and risk of coronary heart disease, stroke and mortality : an individual participant metaanalysis. Lancet. 2010; 375: (9709): 132-140. doi: 10.1016/S01406736(09)61717-7.

5. Tsimikas S, Hall JL. Lipoprotein (a) as a potential causal genetic risk factor of cardiovascular disease: a rationale for increased efforts to understand its pathophysiology and develop targeted therapies. J Am CollCardiol. 2012; 60(8): 716-721. doi: 10.1016/j.jacc.2012.04.038.

6. Kamstrup PR, Tybjærg-Hansen A, Nordestgaard BG. Extreme lipoprotein (a) levels and improved cardiovascular risk prediction. J Am CollCardiol. 2013; 61(11): 1146-1156. doi: 10.1016/j. jacc.2012.12.023.

7. Lin CP, Chen YH, Lew HB, Lin SJ, Chen YL, Huang SL, et al. Antiinflammatory strategies for homocysteine related cardiovascular disease. Front Biosci. 2009; 14: 3836-3845.

8. Yang Q, Botto LD, Erickson JD, Berry RJ, Sambell C, Johansen H, et al. Improvements in stroke mortality in Canada and the United States 1990 to 2002. Circulation. 2006; 113(10): 1335-1343.

9. Wang S, Ma AQ, Song SW, Quan QH, Zhao XF, Zheng XH. Fish oil supplementation improves large arterial elasticity in overweight hypertensive patients. Eur J ClinNutr. 2008; 62(12): 1426-1431.

10. Ueland PM, Refsum H, Stabber SP, Malinow MR, Andersson A, Allen RH. Total homocystiene in plasma or serum-Methods and clinical applications. Clin. Chem. 1993; 39(9): 1764-1779.

11. Ashfield-Watt PL, Moat SJ, Doshi SN, McDowell IF. Folate, homocystiene, endothelial function and cardiovascular disease. 2001; 55(8): 425-433.

12. Dhonskshe-Rutlen RM, de Vries JH, de Bree A, Vander Put N, van Staveren WA, de Groot LC. Dietary intake and status of folate and vitamin B12 and their association with homocysteine and cardiovascular disease in European populations. Eur J. of Clin Nut. 2009; 63(1): 18-30.

13. Ward M, McNulty H, McPartlin J, Strain JJ, Weir DG, Scott JM. Plasma homocystiene, a risk factor for cardiovascular disease, is lowered by physiological doses of folic acid. QJM. 1997; 90(8): 519-524.

14.Lemasters JJ. Selective Mitochondrial Autophagy, or Mitophagy, as a Targeted Defense Against Oxidative Stress, Mitochondrial Dysfunction, and Aging. Rejuvenation Research. 2005; 8(1): 3-5.

15. Stanner SA, Hughes J Kelly CN, Buttriss J. A review of the epidemiological evidence for the antioxidant hypothesis. Public Health Nutrition. 2004; 7(3): 407-422. 
16. Cordoba JM, Martinez-Gonzalez MA. Antioxidant vitamins and cardiovascular disease. Curr Top Med Chem; 2011; 11(14): 18611869.

17. Houston MC. The role of cellular micronutrient analysis, nutraceuticals, vitamins, antioxidants and minerals in the prevention and treatment of hypertension and cardiovascular disease. TherAdv Cardiovascular Disease; 2010; 4(3): 165-183. doi: 10.1177/1753944710368205.

18. Buijsse B, Weikert C, Drogan D, Bergmann M, Boeing H. Chocolate consumption in relation to blood pressure and risk of cardiovascular disease in German adults. Eur Heart Journal. 2010; 31(13): 16161623. doi: 10.1093/eurheartj/ehq068.

19. Littarru GP, Lippa S, Oradie S, Fiorni RM, Mazzanti L. Metabolic and diagnostic implications of blood CoQ10 levels. Biomedical and Clinical Aspects of Coenzyme Q10.1991; 6: 167-178.

20. Littarru GP, Cadenas E, Packer L. Clinical aspects of Coenzyme Q10: Improvement of cellular bioenergetics or antioxidant protection? In: Cadenas E, Packer L, editors. Handbook of Antioxidants. Newyork: Marcell Dekker, Inc. 1996; 203-239.

21. Langsjoen $P$, Langsjoen $P$, Willis R, Folkers K. Treatment of essential hypertension with Coenzyme Q10. Holic Aspects Med. 1994; 15: 265 272.

22. Steven DE. Coenzyme Q10. Phoenix AZ. Review provided by Veri Med Health Care Network. 2009.

23. Murray MT. Encyclopaedia of Nutritional Supplements. Rocklin: Prema Publishing; 1996; 296-308.

24.Langsjoen PH, S Vadhanavikit, K Folkers. Response of patients in classes III and IV of cardiomyopathy to therapy in a blind and crossover trial with Coenzyme Q10. Proceedings of the National Academy of Sciences USA. 1985; 82(12): 4240-4244.

25. Steven G, Frishman WH. Coenzyme Q10: A new drug of cardiovascular disease. Journal of Clinical Pharmacology. 1990; 30(7): 596-608.

26. Fuke C, Krikorian SA, Couris RR. Coenzyme Q10: a review of essential function and clinical trials. US Pharmacist. 2000; 28-41.

27. Trinder P. Determination of Glucose in blood using glucose oxidase with alternate oxygen acceptor. Am Clin Biochem. 1969; 6: 24-27.

28. Roescheau P, Bernt E, Gruber WA. (1974) Clinical Chemistry. Clin Biochem. 12: 226

29. Fossati P, Prencipe L. Serum triglycerides determined colorimetrically with an enzyme that produces hydrogen peroxide. Clin Chem. 1982 ; 28: 2077-2080.

30. Castelli WP. HDL Cholesterol and other lipids in coronary heart disease. Circulation. 1977; 55: 767-772.

31. ICMR. Nutrient Requirement and recommended dietary allowances for Indian. A Report of the Expert Group of the ICMR, New Delhi. 2010.

32. ICMR. Guidelines for Institutional Ethical Committee, New Delhi. 2008.

33. Sharma JK. Business Statistics. 2nd edn. New Delhi: Pearson Education India; 2008.

34. Marinou K, Tousoulis D, Antonopoulos AS, Stefanadi E, Stefanadis C. Obesity and cardiovascular disease: From pathophysiology to risk stratification. Int J of Cardiol. 2010; 138(1): 3-8. doi: 10.1016/j. ijcard.2009.03.135.

35. McCully KS. Vascular pathology of homocyst(e)inemia: Implication for the pathogenesis of arteriosclerosis. Am J Pathol. 1969; 56(1): 111128.

36. Hausenloy DJ and Yellon DM. Enhancing cardiovascular disease risk reduction: raising high density lipoprotein levels. Current Opinion in Cardiology. 2009; 24(5): 473-482. doi: 10.1097/ HC0.0b013e32832ebfe7.

37. Estruch R, Martinez-Gonzalez MA, Corella D, Basora-Gallisa J, Ruiz-Gutierrez V, Convas MI, et al. Effects of dietary fiber intake on risk factors for cardiovascular disease in subjects at high risk. J Epidemiol Community Health. 2009; 63(7): 582-588. doi: 10.1136/ jech.2008.082214. 